\title{
EDITORIAL
}

\section{Does Ibuprofen Prevent Acute Mountain Sickness?}

Two recent prospective double-blind, placebo controlled studies, one by Lipman et al in the Annals of Emergency Medicine ${ }^{1}$ and another by Gertsch et al in this issue of Wilderness \& Environmental Medicine, ${ }^{2}$ have evaluated the use of ibuprofen, for prevention of acute mountain sickness (AMS). These studies raise the questions of whether ibuprofen is useful to prevent AMS and, if so, how does it work? Because the current diagnostic criteria for AMS require the presence of a headache, does ibuprofen prevent AMS by preventing the headache without providing protection against the other features of the disease? Does ibuprofen speed acclimatization or does it mask the symptoms of AMS?

Acute mountain sickness is a symptom complex that afflicts visitors to high altitude, generally above $2500 \mathrm{~m} .^{3}$ Acute mountain sickness is unpleasant and is usually self-limited, but can progress to high altitude cerebral edema (HACE), a life-threatening illness. The Lake Louise Acute Mountain Sickness Scoring System-Lake Louise Score (LLS) for short-is the most commonly used method of diagnosing AMS. The LLS bases the diagnosis of AMS on "a recent gain in altitude, at least several hours at the new altitude and the presence of a headache, and at least one of the following symptoms: gastrointestinal upset (anorexia, nausea, or vomiting), fatigue or weakness, dizziness or lightheadedness, and difficulty sleeping." (Table) A score of 3 points or more, with at least 1 of the points as a result of headache, establishes the diagnosis of AMS.

There are several ways to prevent AMS when going to high altitude. The most reliable and safest method is gradual ascent to allow time for acclimatization. Two medications - acetazolamide and dexamethasone- have been shown to decrease the risk of AMS when given prophylactically. ${ }^{5}$ An herbal medication, Ginkgo biloba, has also been studied for AMS prevention, with mixed results. ${ }^{5}$ Individual double-blind placebo-controlled studies have reported reduction in the incidence of AMS with the use of gabapentin ${ }^{6}$ or sumatriptan. ${ }^{7}$

In the first of the recent studies, Lipman et $\mathrm{al}^{1}$ studied AMS in subjects who ascended rapidly from below $1240 \mathrm{~m}$ to $3810 \mathrm{~m}$ in the White Mountains of California. The authors concluded that ibuprofen was effective when compared with placebo in reducing the incidence of
AMS. In the second, Gertsch et $\mathrm{al}^{2}$ studied AMS in the Nepal Himalayas in trekkers who walked from about $4300 \mathrm{~m}$ to $4928 \mathrm{~m}$. The authors found that ibuprofen was "effective in preventing AMS in the intent-to-treat analysis group, but not in those who completed the trial." They suggested that subjects in the placebo group were more likely to experience AMS and therefore to drop out of the trial. As with many previous studies in the Everest region, the sample size was large, but the rate of noncompletion was also relatively high.

Is the apparent benefit of ibuprofen in preventing AMS based only on its ability to prevent headache? Because headache is an essential symptom in the current diagnosis of AMS, the efficacy of ibuprofen may be an artifact of the scoring system. A recent debate over the merits of changing the LLS to eliminate that requirement that a headache be present underscores the uncertainty of diagnosis. West ${ }^{8}$ argued that there are many people who suffer from acute altitude-related symptoms but do not have a headache, whereas Roach et $\mathrm{al}^{9}$ maintained that the presence of headache was important, at least for research purposes.

Complicating this question is the existence of another entity, high altitude headache (HAH), a necessary but not sufficient condition for AMS using the LLS. In the Headache Evaluation at Altitude Trial (HEAT), ${ }^{10}$ acetazolamide and ibuprofen had similar efficacy in preventing HAH and both were more effective than placebo. As with AMS, the differential diagnosis of headache at high altitude is broad. Both AMS and HAH can be simulated by other conditions, such as migraine and carbon monoxide poisoning, that are not necessarily related to altitude exposure.

The authors of both recent studies cite the importance of inflammation in the pathophysiology of $\mathrm{AMS}^{1-13}$ as supporting evidence that a nonsteroidal anti-inflammatory drug (NSAID) such as ibuprofen should be of benefit in preventing AMS. The study by Lipman et $\mathrm{al}^{1}$ showed a similar prevalence of headache in the AMS and placebo groups. As the authors point out, this favors the conclusion that ibuprofen acts to prevent the constellation of symptoms rather than just treating the headache. Burtscher et al in a small, randomized, double-blind, placebo-controlled trial ${ }^{14}$ found that aspirin helped to 
prevent HAH. The authors theorized that aspirin "may support adaptation to high altitude by reducing sympathetic activity mediated by prostaglandin." In contrast, a small study of the NSAID calcium carbasalate, an aspirin analog, failed to show any preventive effect against AMS compared with placebo. ${ }^{15}$ This study, on Mt Kilimanjaro, was limited by a very rapid ascent rate, but acetazolamide did provide significant protection in the study. A study comparing paracetamol (acetaminophen) with ibuprofen for the prevention of AMS might shed light on the relative importance of antiheadache and anti-inflammatory effects because paracetamol has no significant anti-inflammatory effect but is effective against headache.

Does ibuprofen speed acclimatization or does it just mask symptoms? One answer for an anti-inflammatory medication that is not an NSAID comes from a hypobaric chamber study of the use of dexamethasone to prevent AMS in 6 subjects. ${ }^{16}$ Although dexamethasone was effective in preventing AMS, the authors found no improvement in objective physiologic abnormalities related to hypoxia. Alarmingly, they identified cerebral edema in 1 subject. They concluded that dexamethasone works to prevent AMS by mood enhancement and by decreasing nausea. Withdrawal of dexamethasone after rapid ascent to high altitude, without sufficient time for acclimatization, can cause "rebound" symptoms. Further ascent after taking dexamethasone for prevention or treatment of AMS carries a greater risk of AMS or HACE than does further ascent after use of acetazolamide, which speeds acclimatization. The usual explanation has been that dexamethasone "masks" symptoms of AMS.

Ibuprofen is a relatively benign drug, although its use does carry potentially serious risks such as gastrointestinal hemorrhage. Ibuprofen tends to have fewer side effects than acetazolamide. Unlike dexamethasone, it does not cause euphoria or decrease nausea. Its analgesic and anti-headache properties are limited and unlikely to mask significant symptoms. The widespread popularity of ibuprofen and the fact that it is widely available without a prescription make it an attractive option for prevention of AMS if the early reports of its effectiveness prove to be true.

One could speculate that travelers to high altitude who are taking ibuprofen for prevention of AMS, like those taking dexamethasone, should be cautious not to discontinue it too soon and should also be cautious about continued ascent. However, there is no evidence to suggest that this is the case and no reports of problems to suggest that these precautions are necessary.

Current evidence suggests that ibuprofen is effective in the prevention of AMS and that its benefit is not limited to preventing headache. It is likely that ibuprofen acts to protect against AMS by decreasing inflammatory responses to hypoxia. Whether ibuprofen masks symptoms or speeds acclimatization is still an open question. The bright side of these questions is that there is ample scope for further research!

Ken Zafren, MD

Department of Emergency Medicine Alaska Native Medical Center, Anchorage, AK Division of Emergency Medicine Stanford University Medical Center, Palo Alto, CA Himalayan Rescue Association Kathmandu, Nepal

\section{References}

1. Lipman GS, Kanaan NC, Holck PS, Constance BB, Gertsch JH; PAINS Group. Ibuprofen prevents altitude illness: a randomized controlled trial for prevention of altitude illness with nonsteroidal anti-inflammatories. Ann Emerg Med. 2012;59:484-490.

2. Gertsch JH, Corbett B, Holck PS, et al. Altitude Sickness in Climbers and Efficacy of NSAIDs Trial (ASCENT): randomized, controlled trial of ibuprofen versus placebo for prevention of altitude illness. Wilderness Environ Med. 2012;23:307-315.

3. Hackett PH, Roach RC. High-altitude illness. $N$ Engl J Med. 2001;345:107-114.

4. Roach R, Bärtsch P, Oelz O, Hackett PH. The Lake Louise acute mountain sickness scoring system. In: Sutton JR, Houston CS, Coates G, eds. Hypoxia and Molecular Medicine. Burlington, VT: Queen City Printers; 1993:272-274.

5. Luks AM, McIntosh SE, Grissom CK, et al. Wilderness Medical Society consensus guidelines for the prevention and treatment of acute altitude illness. Wilderness Environ Med. 2010;21:146-155.

6. Jafarian S, Gorouhi F, Salimi S, Lotfi J. Low-dose gabapentin in treatment of high-altitude headache. Cephalalgia. 2007;27:1274-1277.

7. Jafarian S, Gorouhi F, Salimi S, Lotfi J. Sumatriptan for prevention of acute mountain sickness: randomized clinical trial. Ann Neurol. 2007;62:273-277.

8. West JB. Con: headache should not be a required symptom for the diagnosis of acute mountain sickness. High Alt Med Biol. 2011;12:23-25; discussion 27.

9. Roach R, Kayser B, Hackett P. Pro: headache should be a required symptom for the diagnosis of acute mountain sickness. High Alt Med Biol. 2011;12:21-22; discussion 29.

10. Gertsch JH, Lipman GS, Holck PS, et al. Prospective, double-blind, randomized, placebo-controlled comparison of acetazolamide versus ibuprofen for prophylaxis against high altitude headache: the Headache Evaluation at Altitude Trial (HEAT). Wilderness Environ Med. 2010;21: 236-243. 
11. Richalet JP, Hornych A, Rathat C, Aumont J, Larmignat P, Rémy P. Plasma prostaglandins, leukotrienes and thromboxane in acute high altitude hypoxia. Respir Physiol. 1991;85:205-215.

12. Tissot van Patot MC, Leadbetter G, Keyes LE, et al. Greater free plasma VEGF and lower soluble VEGF receptor-1 in acute mountain sickness. J Appl Physiol. 2005; 98:1626-1629.

13. Julian CG, Subudhi AW, Wilson MJ, Dimmen AC, Pecha T, Roach RC. Acute mountain sickness, inflammation, and permeability: new insights from a blood biomarker study. J Appl Physiol. 2011;111:392-399.
14. Burtscher M, Likar R, Nachbauer W, Philadelphy M. Aspirin for prophylaxis against headache at high altitudes: randomised, double blind, placebo controlled trial. BMJ. 1998;316:1057-1058.

15. Kayser B, Hulsebosch R, Bosch F. Low-dose acetylsalicylic acid analog and acetazolamide for prevention of acute mountain sickness: the authors reply. High Alt Med Biol. 2008;9:351-352.

16. Levine BD, Yoshimura K, Kobayashi T, Fukushima M, Shibamoto T, Ueda G. Dexamethasone in the treatment of acute mountain sickness. N Engl J Med. 1989;321: 1707-1713. 